\title{
EXPRESSION OF A-, B-, AND TENAC IN HUMAN FETAL AND NEWBORN LUNG
}

C. Janér ${ }^{1,2}$, R. Karikoski ${ }^{3}$, O. Helve ${ }^{4}$, J. Janér ${ }^{5}$, A. Yli-Peltola ${ }^{1}$, C. Haglund ${ }^{6}$, O.M. Pitkänen ${ }^{4}$, S. Andersson ${ }^{4}$

${ }^{1}$ Pediatric Research Center, Children's Hospital, Helsinki University Central Hospital, ${ }^{2}$ Clinical Graduate School in Pediatrics and Obstetrics/Gynecology, University of Helsinki, ${ }^{3}$ Department of Pathology, Helsinki University Central Hospital, ${ }^{4}$ Children's Hospital, Helsinki University Central Hospital, ${ }^{5}$ Department of Pediatrics, ${ }^{6}$ Department of Surgery, Helsinki University Central Hospital, Helsinki, Finland

Background: Airway epithelial sodium channel $\mathrm{ENaC}$ is critical to perinatal lung fluid clearance. Data on $\mathrm{ENaC}$ protein expression in developing human lung are limited and absent for infants with RDS or BPD.

Objective: To characterize the expression of $\alpha-, \beta$-, and $\gamma \mathrm{ENaC}$ expression in lung tissue from human fetuses and newborns with and without RDS or BPD.

Methods: Lung tissue was obtained from autopsies $(\mathrm{n}=23)$ from fetuses (GA $12-21$ wks), preterm infants with (GA 25 - 28 wks) or without RDS (GA 23 - 25 wks), and infants with BPD (GA 24 - 30 wks at birth). The primary antibodies were rabbit polyclonal $\alpha \mathrm{ENaC}$ (1:60 Sigma-Aldrich Inc, MO, US), $\beta E N a C$ (1:2250 Aviva Systems Biology, CA, US) or $\gamma \mathrm{ENaC}$ (1:200 Lifespan Biosciences Inc, WA, US).

Results: In fetal lung developing respiratory, glandular epithelium stained with $\alpha-, \beta-$, and $\gamma \mathrm{ENaC}$ from $12+3$ wks GA. In preterm infants without RDS expression of $\alpha-, \beta$-, and $\gamma \mathrm{ENaC}$ was apparent in single type II pneumocytes, while type I pneumocytes remained unstained. In RDS and BPD the expression of ENaC subunits in alveolar epithelium was largely absent but reactive, hyperplastic type II pneumocytes stained strongly for $\alpha$ - and $\beta \mathrm{ENaC}$. Throughout development, and in infants with RDS or BPD $\alpha-, \beta-$, and $\gamma \mathrm{ENaC}$ stained the bronchiolar epithelium.

Conclusions: In human fetal lung all three $\mathrm{ENaC}$ subunits were expressed during the pseudoglandular stage of lung development. Expression of $\mathrm{ENaC}$ subunits in bronchioles may indicate a role for conductive airways in perinatal lung liquid clearance. 